\title{
Conformity of removable partial denture designs to agreed principles based on materials used
} - A preliminary study

SADJ June 2020, Vol. 75 No. 5 p253 - p259

W Farao', G Geerts ${ }^{2}$

\section{ABSTRACT \\ Introduction \\ Removable partial denture (RPD) designs may differ based on types of materials used.}

\section{Aims}

The aim of the study was to investigate how a sample of non-metal clasp (NMC), acrylic and metal RPD designs complied with biomechanical design principles.

\section{Methods}

This cross-sectional study examined 60 clinical designs of NMC, acrylic and metal RPDs at 3 commercial dental laboratories in the Cape Town Metropole, at the stage when the dentures were ready for transport to dentists. Retention, indirect retention, support, soft tissue cover, and cross-arch design features were recorded and compared with "ideal" control designs developed by 2 experts in the specialty of prosthodontics.

\section{Results}

Fifty five \% of the clinical designs had no rests, hence were mucosa supported. None of the NMC and only $35 \%$ of acrylic RPDs had some rests. A total of 35 clinical designs required indirect retention, but it was only provided in 14 (40\%) of them. Eighty five \% of acrylic RPDs had no clasps; metal RPDs had more clasps than required while NMC RPDs had slightly less clasps than required.

\section{Author affiliations:}

1. Warren Farao: $B C h D, P D D, M C h D$, Senior Lecturer, Specialist: Prosthodontist, Faculty of Dentistry, Tygerberg Campus, University of the Western Cape, Tygerberg Oral Health Centre, Tygerberg, South Africa.

ORCID Number: 0000-0002-1327-8170

2. Greta Geerts: $B C h D, P D D, M C h D, P h D$, Senior lecturer Chie specialist, Faculty of Dentistry, Tygerberg Campus, University of the Western Cape, Tygerberg Oral Health Centre, Tygerberg, South Africa.

ORCID Number: 0000-0002-6324-4251

Corresponding author: Warren Farao

Senior Lecturer, Specialist: Prosthodontist, Faculty of Dentistry,

Tygerberg Campus, University of the Western Cape, Tygerberg Oral

Health Centre, Tygerberg, South Africa.

Email: wfarao@uwc.ac.za

Author contributions:

1. Warren Farao: Principal researcher - $75 \%$, Writing article - $25 \%$

2. Greta Geerts: Research - $25 \%$, Writing article $-75 \%$,
Ratio teeth covered/replaced was most favourable for metal (0.91), followed by acrylic (1.83) and NMC (1.80) RPDs. Cross - arch stabilization was absent in $22 \%$ of clinical designs, all of them from the NMC group.

\section{Conclusion}

Of the 3 types of RPDs, metal RPDs complied best with requirements in terms of tissue support (mostly tooth and mixed tooth/mucosa), retention, cross-arch stabilization and "open" design. Acrylic RPDs provided crossarch stabilization but were lacking in all other aspects. Except for direct retention, NMC RPDs did not conform to any of the agreed biomechanical requirements for RPDs assessed in this study.

\section{INTRODUCTION}

In industrialised countries, edentulism decreased due to improved oral hygiene, patient health and dental care, with many people retaining some of their natural dentition into old age, and an increased desire by patients to retain their teeth. ${ }^{1}$ A shift from total to partial edentulism, has led to a greater need to replace missing teeth for partially edentulous patients. ${ }^{1}$

One of the options for tooth replacement in partially edentulous patients is RPDs. Removable partial dentures have to comply with biomechanical aspects of support, retention and stability as well as limit damage to intraoral hard and soft tissues. ${ }^{2-5}$

The Academy of Prosthodontics publishes and regularly updates its principles, concepts, and practices in prosthodontics, including those for RPDs based on feedback from members and working committees of several Academies. ${ }^{6}$ However, there appear to be variations in how these principles and concepts are applied. ${ }^{7}$ In addition, there is little evidence that adhering to these principles leads to improved treatment outcomes or patient satisfaction. ${ }^{8}$

Many studies have reported on the adverse effects of RPDs on oral tissues. They may be associated with higher plaque (PI), gingival (GI) and calculus indices (Cl), increased probing depth (PD), gingival recession, root caries and mobility of abutment teeth. ${ }^{9-16}$ However, with 
high level of patient cooperation and motivation, the number of teeth lost, decayed and filled due to RPD wearing, can be limited. Wilson (2009) proposed that acrylic RPDs can be considered definitive prostheses provided that proper patient selection and design principles are followed. ${ }^{17}$ Hence, it is the clinician's responsibility to plan and design each RPD in such a way that the balance between benefits and risks of placing a RPD is in the patient's favour. ${ }^{18}$

Several materials may be used to fabricate RPDs. Cobalt chromium alloys and polymethyl methacrylate resin are the traditional materials. Newer materials, known as "thermoplastic" or "flexible" materials have been developed. These materials include a variety of polymers: polyethylene glycol, methyl methacrylate, aryl-ketone polymers, polyamide resins, polycarbonate resins, polyethylene terephthalate resins. ${ }^{19}$ Dentures fabricated from these materials are referred to as non-metal clasp (NMC) dentures. ${ }^{19}$ Physical properties vary considerably among of this group of new materials. ${ }^{20,21}$

It is claimed that NMC RPDs lead to improved patient comfort and satisfaction compared to acrylic RPDs, blend better with oral tissues hence are aesthetically more pleasing than some of the traditional materials and are hypo-allergenic. ${ }^{22}$ Even though long-term evidence and guidelines for the use of NMC RPDs has been lacking, they attracted interest from practitioners.

This led to the development of a "position paper" based on "expert opinion" by the Japan Prosthodontic Society, wherein NMC RPDs were not recommended as defini tive prostheses, except in case such as metal allergy or when rigidity can be provided by incorporating a rigid framework. ${ }^{19,20,23}$ A major disadvantage of NMC RPDs includes clasps covering the cervical area of the tooth, marginal gingiva and mucosa - as opposed to metal clasps which do not come into contact with the gingival margin - hence may cause caries and periodontal disease. ${ }^{20}$ In addition, if NMC RPDs do not have rigid rests and frameworks, their clasps can traumatize marginal gingiva. ${ }^{20}$

It has been the impression of the authors that application of RPD design principles differ based on the types of materials used. Therefore, the aim of the study was to assess how a sample of clinical NMC, acrylic and metal RPDs comply with accepted biological and mechanical design principles. The null-hypothesis was that there is no difference among RPDs made from different materials in complying with biological and mechanical design principles.

\section{MATERIALS AND METHODS}

The research proposal was approved by the biomedical research ethics committee of the University of the Western Cape (BM/16/5/12). The project is a cross-sectional study using a convenience sample. Three dental laboratories in the Cape Town Metropole, known to fabricate NMC RPDs, were identified and invited to participate in the study. Their participation required of them to email photographs of completed NMC, acrylic and metal RPDs and their casts prior to sending them to practices for delivery to patients. The following views of the RPDs were requested: occlusal, left lateral, right lateral, frontal and any other view to enable the researchers to identify all RPD components on the photographs. Specimens were collected until 20 metal, 20 acrylic and 20 NMC RPDs were received. Data collection took place during 2018.

For each RPD, the actual design as well as an "ideal" design was drawn, using the image of the cast. This was done independently by two observers, who are experienced members of staff in the Department of Restorative Dentistry (Prosthetics).

The ideal designs from both observers were later compared for similarity. Differences in designs were resolved by means of discussion until agreement was reached. Each ideal design served as the control for its clinical design.

Prior to designing the ideal RPDs, for the sake of consistency, the following agreements were made:

- Mandibular lingual major connector for control design was kept the same as the clinical RPD design (plate vs. bar).

- Number of replaced teeth on ideal denture was kept the same as number of teeth replaced on the clinical RPDs.

Prior to recording data from the RPD and ideal designs, for the sake of consistency, the following agreements were made:

- The number of rests was counted and was given as a numerical value: 1, 2, 3, 4, >4. The configuration of rests was given as 0 : no configuration because there were no rests or only one rest; 2 : line; 3 : triangle; 4 : at least a quadrangle.

- Type of support for the RPD was indicated as: hard (exclusively tooth-born), soft (exclusively mucosa-born) or mixed (both tooth and mucosa support).

- Number of clasps was counted and was given as a numerical value: 1, 2, 3, 4, >4.

- Presence of indirect retention was given as: yes, no or not applicable. An indirect retainer helps to stabilize a distal extension RPD. The requirement of indirect retention for Kennedy Class III and IV were also identified where applicable. The indirect retention component is usually located on the opposite side of the retentive fulcrum line from the mucosa supported base. ${ }^{24}$

- Number of teeth with gingival margin cover as well as number of replaced teeth were given as a numerical value: $1,2,3 \ldots$ These values were used to match replaced/covered teeth to calculate a "coverage" score to give an indication of a biological price to tissue.

- Presence of cross-arch stability: yes or no. A 'no' was allocated to a unilateral RPD. A unilateral RPD is sometimes used to restore short-span, bounded edentulous spaces with the advantage of avoiding 
extensive palatal or lingual major connectors. This design may result in easy dislodgement and other complications because it does not provide crossarch stabilization. RPDs with cross-arch stabilization are generally preferred to prevent these complications. ${ }^{25}$

Compliance to the ideal design was rated as 'Acceptable' or 'Not acceptable' according to the following general rules:

- For support, for Class II, III and IV RPDs a minimum of 3 rests in a triangle configuration was considered acceptable, less than 3 rests or 3 rests not in a triangle configuration was considered not acceptable. For Class I RPDs a minimum of 2 rests was considered acceptable, less than 2 rests was considered not acceptable.

Soft tissue support was not acceptable for any of the classifications. Mixed support was accepted for Class I, II and other classifications where long saddles covered arch bends; for all other designs, only hard support was acceptable.

- Absence of indirect retention for designs where it was indicated was scored as not acceptable.

- Absence of cross-arch stability was scored as not acceptable.

Two observers rated acceptability independently. Where differences occurred, these were debated until consensus was reached. Results are presented descriptively using frequency and ratio tables. Because of the nature of the data, statistical analysis was not indicated.

\section{RESULTS}

The features of all clinical and control designs are summarized in Table 1 . The majority of the 60 RPDs were for the maxilla $(n=42 ; 70 \%)$. Kennedy Class III designs occurred most frequently with a total of 44 (73\%) RPDs. There was only one Class IV design, belonging to the acrylic RPD group.

Thirty-three (55\%) clinical designs had no rests, 8 (13\%) had only 1 rest, 8 (13\%) had 2 rests, 4 (7\%) had 3 rests, and $7(12 \%)$ had 4 or more rests. All NMC RPDs (100\%) had no rests at all. The control designs all had rests, with 57 (95\%) designs having at least 3 rests. There were no control designs with 1 or zero rests (Table 1 ).

A total of 33 clinical designs were mucosa supported (Acrylic clinical=13, Metal clinical $=0, N M C$ clinical $=20$ ), 21 were of mixed support (Acrylic clinical $=6$, Metal clinical 15, NMC clinical $=0$ ) and 6 had hard tissue support (Acrylic clinical $=1$, Metal clinical $=5$, NMC clinical $=0)$.

All the control designs had either hard tissue $(n=45)$ or mixed support $(n=15)$ (Table 1).

Total and mean number of clasps for each RPD group, clinical and control, are shown in Table 2. The clinical acrylic and NMC RPDs had fewer clasps than their controls. Seventeen (85\%) of clinical acrylic RPDs had no clasps at all.

A total of 35 designs (including distal extension bases and maxillary Class IV dentures) required indirect retention and in only 14 designs indirect retention was provided (40\% compliance rate). (Figure 1) Compliance rate per RPD materials group was as follows: metal $83.33 \%$, acrylic 6.25\%, NMC 42.86\%.

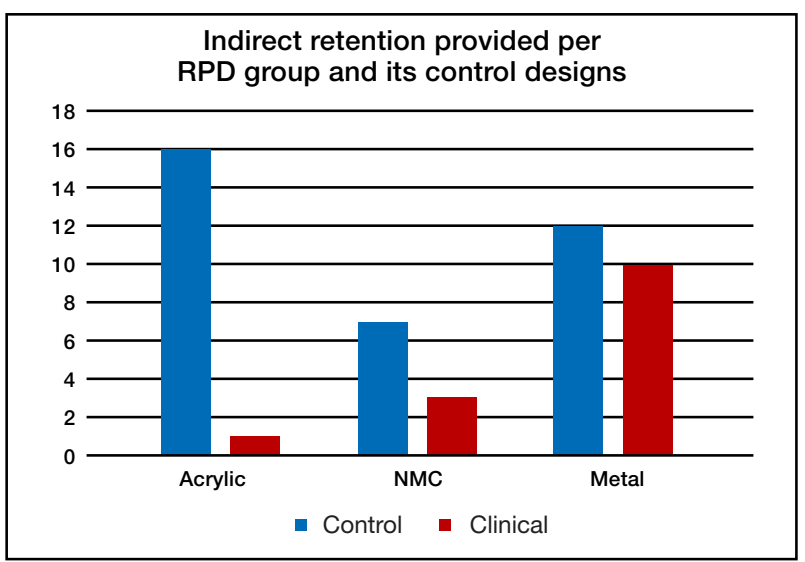

Figure 1. Indirect retention provided per RPD group and its control designs.

The number of teeth whose gingival tissue was covered versus the number of teeth replaced is shown in Table 1 and the ratios are shown in Table 4.

All control designs had cross-arch stabilization, as had the acrylic and metal groups. The NMC group only had 7 out of 20 designs with cross-arch stabilization (Table 1). Acceptability of designs according to mechanical and biological principles is shown in Table 4.

\section{DISCUSSION}

Differences in compliance with biological and mechanical principles were found among designs of RPDs made from different base materials. Therefore, the null-hypothesis is rejected.

The rejection of the null-hypothesis implies that there is disparity between the teaching of generally accepted RPD design principles according to the literature and what is happening in private practice. A survey on a group of dentists that graduated at the dental school of the University of the Witwatersrand alluded to a similar situation. ${ }^{26}$

At the University of the Western Cape open, hygienic designs, an appropriate number of clasps and rests, and other essential RPD components to ensure adequate support, retention and stability, are taught for RPDs.

This survey showed that the disparity between design principles and clinical practice was generally smaller for metal, and larger for acrylic and NMC RPDs. This may be, to some extent, due to limitations of the material and not to a gap between knowledge and practice. In addition, NMC RPDs are not taught at the dental school of the UWC. 


\begin{tabular}{|c|c|c|c|c|c|c|}
\hline & \multicolumn{2}{|c|}{ Acrylic } & \multicolumn{2}{|c|}{ Metal } & \multicolumn{2}{|c|}{ NMC } \\
\hline & Clinical & Control & Clinical & Control & Clinical & Control \\
\hline Number & 20 & 20 & 20 & 20 & 20 & 20 \\
\hline Class I & 3 & 3 & 2 & 2 & 1 & 1 \\
\hline Class II & 2 & 2 & 4 & 4 & 3 & 3 \\
\hline Class III & 14 & 14 & 14 & 14 & 16 & 16 \\
\hline Class IV & 1 & 1 & 0 & 0 & 0 & 0 \\
\hline Mandibular & 5 & 5 & 9 & 9 & 4 & 4 \\
\hline Maxillary & 15 & 15 & 11 & 11 & 16 & 16 \\
\hline Total no. of rests & 12 & 88 & 57 & 78 & 0 & 80 \\
\hline No of RPDs without rests & 13 & 0 & 0 & 0 & 20 & 0 \\
\hline RPDs with 1 rest & 6 & 0 & 2 & 0 & 0 & 0 \\
\hline RPDs with 2 rests & 0 & 1 & 8 & 1 & 0 & 1 \\
\hline RPDs with 3 rests & 0 & 1 & 4 & 4 & 0 & 2 \\
\hline RPDs with 4 or more rests & 1 & 18 & 6 & 15 & 0 & 17 \\
\hline RPDs with no rest configuration & 19 & 0 & 2 & 0 & 20 & 0 \\
\hline RPDs configuration in line & 0 & 1 & 8 & 1 & 0 & 1 \\
\hline RPDs configuration of rests in triangle & 0 & 1 & 4 & 4 & 0 & 2 \\
\hline RPDs configuration of rests in square or more & 1 & 18 & 6 & 15 & 0 & 17 \\
\hline Mucosa support & 13 & 0 & 0 & 0 & 20 & 0 \\
\hline Mixed tooth/mucosa support & 6 & 5 & 15 & 6 & 0 & 4 \\
\hline Tooth support & 1 & 15 & 5 & 14 & 0 & 16 \\
\hline Total no. of clasps & 6 & 51 & 71 & 64 & 43 & 52 \\
\hline RPDs without clasps & 17 & 0 & 0 & 0 & 0 & 0 \\
\hline RPDs with 1 clasp & 0 & 0 & 0 & 0 & 1 & 0 \\
\hline RPDs with 2 clasps & 3 & 11 & 2 & 4 & 16 & 9 \\
\hline RPDs with 3 clasps & 0 & 7 & 5 & 8 & 2 & 10 \\
\hline RPDs with 4 clasps & 0 & 2 & 13 & 8 & 1 & 1 \\
\hline Cross-arch stabilization present & 20 & 20 & 20 & 20 & 7 & 20 \\
\hline Number of teeth covered (replaced) & $159(87)$ & $63(87)$ & $85(93)$ & $61(93)$ & $81(45)$ & $61(45)$ \\
\hline No of teeth covered \& (replaced) Class I & $19(21)$ & $16(21)$ & $9(10)$ & $9(10)$ & $8(5)$ & $8(5)$ \\
\hline No of teeth covered \& (replaced) Class II & $12(12)$ & $5(12)$ & $13(19)$ & $13(19)$ & $24(12)$ & $20(12)$ \\
\hline No of teeth covered \& (replaced) Class III & $120(50)$ & $42(50)$ & $63(64)$ & $39(64)$ & $49(28)$ & $33(28)$ \\
\hline No of teeth covered \& (replaced) Class V & $8(4)$ & $0(4)$ & - & - & - & - \\
\hline
\end{tabular}

Table 2. Number of clasps in clinical and control designs.

\begin{tabular}{|c|c|c|c|}
\hline Denture group & Total number of designs & $\begin{array}{l}\text { Clinical designs } \\
\text { - Total number of clasps (mean) }\end{array}$ & $\begin{array}{l}\text { Control designs } \\
\text { - Total number of clasps (mean) }\end{array}$ \\
\hline Acrylic & 20 & $6(0.30)$ & $51(2.55)$ \\
\hline NMC & 20 & $43(2.15)$ & $52(2.60)$ \\
\hline Metal & 20 & $71(3.55)$ & $64(3.20)$ \\
\hline
\end{tabular}

\begin{tabular}{|c|c|c|c|c|c|c|}
\hline & $\begin{array}{l}\text { Acrylic } \\
\text { (Control) }\end{array}$ & Acceptability \% & $\begin{array}{c}\text { NMC } \\
\text { (Control) }\end{array}$ & Acceptability \% & $\begin{array}{c}\text { Metal } \\
\text { (Control) }\end{array}$ & Acceptability \% \\
\hline Number of dentures with rests (Min. 2 rests) & $1(20)$ & $5 \%$ & $0(20)$ & $0 \%$ & $18(20)$ & $90 \%$ \\
\hline Support & $7(20)$ & $35 \%$ & $0(20)$ & $0 \%$ & $20(20)$ & $100 \%$ \\
\hline Rest configurations & $1(20)$ & $5 \%$ & $0(20)$ & $0 \%$ & $20(20)$ & $100 \%$ \\
\hline Indirect retention & $1(16)$ & $6.25 \%$ & $3(7)$ & $42.86 \%$ & $10(12)$ & $83.33 \%$ \\
\hline Direct retention & $3(20)$ & $15 \%$ & $9(20)$ & $45 \%$ & $20(20)$ & $100 \%$ \\
\hline Teeth covered & $159(71)$ & $\begin{array}{c}92 \text { extra } \\
\text { teeth covered }\end{array}$ & $81(63)$ & $\begin{array}{c}18 \text { extra } \\
\text { teeth covered }\end{array}$ & $85(71)$ & $\begin{array}{c}14 \text { extra } \\
\text { teeth covered }\end{array}$ \\
\hline Ratio teeth covered/teeth replaced & $1.83(0.72)$ & - & $1.8(1.36)$ & - & $0.91(0.66)$ & - \\
\hline No. of RPDs with acceptable ratios & $3(20)$ & $15 \%$ & $3(20)$ & $15 \%$ & $9(20)$ & $45 \%$ \\
\hline Cross-arch stabilization & $20(20)$ & $100 \%$ & $7(20)$ & $35 \%$ & $20(20)$ & $100 \%$ \\
\hline
\end{tabular}


For this study, coverage of periodontal tissues of the remaining natural teeth by RPD components was used as a benchmark for rating the biological acceptability of each denture. Mean coverage ratios were lowest for metal RPDs. With mean coverage ratios of $<1$ for both clinical and control designs, the metal RPDs replaced more teeth than there were teeth covered by design components.

The difference in the coverage ratio between clinical and control designs was also the lowest of the 3 denture groups. Hence, the biological price for replacing teeth in this group was least. Mean coverage ratio for the clinical acrylic RPDs was the highest.

The difference in coverage ratio between clinical and control designs was also the largest. Hence, in this study, it may be assumed that design principles were not adequately followed and the biological price paid by tissues to have teeth replaced by acrylic RPDs was the highest.

Coverage ratios for clinical and control NMC designs were both high. This may be explained by, that in this study, the NMC RPDs restored short-span edentulous areas. The difference in mean coverage ratio of clinical and control NMC RPDs was low, indicating that, in terms of biological price, the clinical design resembled optimal design conditions.

Metal RPDs scored best in terms of "open" hygienic designs. (Table 3) This was expected, since the strength of the material allows it to be used in thin sections. In terms of "open" designs, acrylic RPDs and NMCDs were rated as unacceptable (Table 4). However, due to material limitations, it may not be possible to improve their coverage ratios substantially. Hence, it may be expected that the use of acrylic or NMC RPDs will have a negative effect on PI, GI, PD and root caries unless patients are carefully selected and follow a strict maintenance routine..$^{9-15}$

Properly placed occlusal rests on abutment teeth minimizes iatrogenic soft tissue damage. ${ }^{27}$ Hence, a minimum number of rests in an appropriate configuration was also considered a benchmark for assessing the biologic acceptability of the designs. Optimal conditions for vertical support differ according to Kennedy classification. Each clinical design was compared with its optimal control design. Within the acrylic RPD group, only one of the clinical designs had enough rests in an appropriate configuration to be judged acceptable (Tables 1 and 4).

All the other acrylic RPDs had either one or no rests, hence, had mucosa or mixed tooth/mucosa support, where it could have been mixed tooth/mucosa or tooth support respectively. Therefore, it was decided that acrylic RPDs designs in this study sample were not acceptable regarding vertical support and they were potentially harmful to the supporting tissue of the remaining natural teeth. None of the NMC RPDs had rests.

The result is that all the NMC RPDs were considered mucosa supported and none of the 20 designs in this group was acceptable regarding support (Table 4).
In contrast with the acrylic and NMC RPDs, none of the metal-frame RPDs were mucosa supported. Fifteen clinical metal RPDs had mixed tooth/mucosa support where there should only have been 6 with mixed support according to the control designs. It was concluded that only $9(45 \%)$ of the metal RPDs were acceptable in terms of vertical support. However, compared to the acrylic and NMC groups, the metal RPD group performed best in complying with the principle of support.

For stability and support, the selection of at least 3 rests, widely spaced, is advised. ${ }^{28}$ For the metal RPD group, the clinical designs complied on 11 of the 20 occasions in terms of number and configuration of rests.

For the remaining 9 designs, there support and stability was not optimized. For the acrylic RPDs, there was only 1 of the 20 designs with more than 3 rests. The other designs had either no or only 1 rest. This would not allow transmission of chewing forces along the long axis of the abutment teeth.

Therefore, it was concluded that the acrylic RPD group did not conform to this bio-mechanical requirement. For the NMC group, none of the 20 designs had any rests. The clinical consequence of these findings is that for the metal and acrylic RPDs, the integrity of the abutment teeth is at risk due to inadequate transfer of occlusal forces along abutment teeth and stability of the RPDs is in the majority of cases not optimized.

Direct or active retainers or clasps are critical components of an RPD, exerting a force on abutment teeth when the prosthesis is lifted from its support in the opposite direction as its path of insertion, hence providing retention for the prosthesis. ${ }^{28}$ More clasp assemblies lead to higher PI and TM scores, hence too many clasps are to be avoided. For the acrylic group, a total of 6 clasps were provided for the clinical designs, as compared to 51 given to the corresponding control designs.

This is a mean of 0.3 clasps per clinical design. For the control, the mean number of clasps per design was 2.6. It was concluded that the provision of direct retention was generally inadequate for acrylic RPDs hence retention would be poor. The NMC group had 43 clasps (mean 2.2 per RPD) compared to the 52 (mean 2.6 per RPD), of the control. Based on these results, it was concluded that in terms of providing retention, most NMC RPDs would be acceptable. For the metal RPDs, 71 clasps were given (mean 3.6) against their control designs of 64 clasps (mean 3.2).

This is the only group where the clinical designs were given more clasps than their control group. While this may have a positive impact on retention, it may have a negative effect on PI and Gl. It should be noted that the presence of guide planes on teeth and guiding surfaces on tooth-bound saddles was not assessed in this study. These features may reduce the need for direct retention ${ }^{29}$, and have a further positive effect on the health of soft and hard tissue. It should also be noted that the type of material, shape and position of the clasps also have an influence on their retentive force. This was not examined in this study. 
Indirect retention prevents tipping of the RPD around a horizontal axis. Where applicable and needed, the presence or absence of indirect retention was recorded for the sample of RPDs in this study. Sixteen of the acrylic RPDs required the incorporation of indirect retention, with only 1 design complying (6.25\%). In the NMC group, 7 needed indirect retention with only 3 complying (42.9\%).

The metal group had 12 designs that needed indirect retention with 10 complying (83.33\%). The metal group had the highest compliance rate. Poor compliance was noted in the acrylic group, followed by the NMC group.

It should be noted that the number of designs requiring indirect retention differed considerably among the three groups. The clinical significance of these findings is that the retention of the majority of the acrylic RPDs and half of the NMC RPDs is compromised by failing to provide acceptable indirect retention.

For cross-arch stabilization, the following findings were made: All the acrylic and metal RPDs had a bilateral design and complied with this design principle. The NMC group had only 7 bilateral designs. Hence, this group was $65 \%$ non-compliant in terms of cross-arch stability. In a unilateral RPD design with no cross-arch stabilization, the denture may tilt and dislodge causing complications such as aspiration or swallowing of the prosthesis. ${ }^{30}$

Biological and biomechanical criteria were identified in the methodology of this study and potential clinical translations made. However, these translations need to be confirmed by means of clinical trials. The impact of the quality of the designs of different materials group was not measured using patient based outcomes. This should be studied further.

The purpose of this study was to identify potential problems in terms of RPD design. Hence, a preliminary survey using a convenience sample was done. This sample might not be representative of the total RPDs delivered in the Western Cape.

Since issues with compliance with accepted biomechanical principles for RPD design were encountered among the different groups under investigation in this pilot study, further investigations can be launched to examine the influence of training, dentist-technician communication including presence or absence of prescriptions of designs by dentists, pricing of the different types of RPDs and third party payment schemes. Material limitations may influence design features. These design limitations may impact on oral tissue health and patient satisfaction. Not all design flaws illustrated in this survey were attributed to materials limitations.

The investigator relied on commercial laboratories to provide the designs; the sample may have been biased based on selection of designs by the laboratory technician. It is assumed that, by human nature, selection was biased towards the "better" designs. The process of designing in terms of communication and identity of the RPD designer (dentist or technician) was not requested in order to reduce selection bias by the technician.

\section{CONCLUSION}

Within the limitations of this pilot study, it may be concluded that:

- The metal RPDs scored better for support, direct and indirect retention, and open design than the acrylic and NMC RPDs. For cross-arch stabilization, metal designs scored equally with the acrylic group and better than the NMC group.

- The acrylic group scored poorly for support, direct and indirect retention and open design.

- The NMC group scored poorly for support, indirect retention, open design and cross-arch stabilization.

Acknowledgement

Dr F Kimmie for advice regarding data analysis.

Declaration

The authors have no conflict of interest in any company, product or services mentioned in the article.

\section{References}

1. Campbell SD, Cooper L, Craddock H, et al. Removable partial dentures: The clinical need for innovation. J Prosthet Dent. 2017; 118: 273-80.

2. Jacobson TE. Periodontal considerations in removable partial denture design. Compendium 1987; 8: 530-4, 536-9.

3. Budtz-Jorgensen E, Bochet G. Alternate framework designs for removable partial dentures. J Prosthet Dent. 1998; 80: 58-66.

4. Owall B, Budtz-Jörgensen E, Davenport J, et al. Removable partial denture design: a need to focus on hygienic principles? Int J Prosthodont. 2002; 15: 371-8.

5. Davenport JC, Hammond P, de Mattos MG. The acquisition and validation of removable partial denture design knowledge. II. Design rules and expert reaction. J Oral Rehabil. 1996; 23: 811-24.

6. Academy of Prosthodontics. Principles, concepts, and practices in prosthodontics. $9^{\text {th }}$ ed. J Prosthet Dent. 1995; 73: 73-94.

7. Kapur KK, Deupree R, Dent RJ, et al. A randomized clinical trial of two basic removable partial denture designs. Part I: Comparisons of five-year success rates and periodontal health. J Prosthet Dent. 1994; 72: 268-82.

8. Frank RP, Brudvik JS, Leroux B, et al. Relationship between the standards of removable partial denture construction, clinical acceptability, and patient satisfaction. J Prosthet Dent. 2000; 83: 521-7.

9. Mojon P, Rentsch A, Budtz-Jørgensen E. Relationship between prosthodontic status, caries, and periodontal disease in a geriatric population. Int J Prosthodont. 1995; 8: 564-71.

10. Yeung AL, Lo EC, Chow TW, et al. Oral health status of patients 5-6 years after placement of cobalt-chromium removable partial dentures. J Oral Rehabil. 2000; 27: 183-9.

11. Zlataric DK, Celebic A, Valentic-Peruzovic M. The effect of removable partial dentures on periodontal health of abutment and non-abutment teeth. J Periodontol. 2002; 73: 137-44.

12. Akaltan F, Kaynak D. An evaluation of the effects of two distal extension removable partial denture designs on tooth stabilization and periodontal health. J Oral Rehabil. 2005; 32: 823-9.

13. Do Amaral BA, Barreto AO, Gomes Seabra E, et al. A clinical follow-up study of the periodontal conditions of RPD abutment and non-abutment teeth. $J$ of Oral Rehabil. 2010; 37: 545-52. 
14. Preshaw PM, Walls AW, Jakubovics NS, et al. Association of removable partial denture use with oral and systemic health. J Dent. 2011; 39: 711-19.

15. Orr S, Linden GJ, Newman HN. The effect of partial denture connectors on gingival health. J Clin Periodontol. 1992; 19: $589-94$.

16. Bergman B, Hugoson A, Olsson CO. A 25 year longitudina study of patients treated with removable partial dentures. J Oral Rehabil. 1995; 22: 595-9.

17. Wilson VJ. Acrylic partial dentures--interim or permanent prostheses? SADJ. 2009; 64: 434, 436-8, 440.

18. Davenport JC, Basker RM, Heath JR, et al. The removable partial denture equation. Br Dent J. 2000; 189: 414-24.

19. Fueki K, Onkubo C, Yatabe M, et al. Clinical application of removable partial dentures using thermoplastic resin-part I: definition and indication of non-metal clasp dentures. J Prosthodont Res. 2014; 58: 3-10.

20. Fueki K, Ohkubo C, Yatabe M, et al. Clinical application of removable partial dentures using thermoplastic resin. Part II: Material properties and clinical features of non-metal clasp dentures. J Prosthodont Res. 2014; 58: 71-84.

21. Takabayashi Y. Characteristics of denture thermoplastic resins for non-metal clasp dentures. Dent Mater J. 2010; 29: 353-61.

22. Singh JP, Dhiman RK, Bedi RP, et al. Flexible denture base material: A viable alternative to conventional acrylic denture base material. Contemp Clin Dent. 2011; 2: 313-7.
23. Fueki K. Non-metal clasp dentures: More evidence is needed for optimal clinical application. J Prosthodont Res. 2016; 60: 227-8.

24. Davenport JC, Basker RM, Heath JR, et al. Indirect retention. Br Dent J. 2001; 190: 128-32.

25. Uludag B and Celik G. Technical tips for improved retention and stabilization of a unilateral removable partial denture. J Oral Impl. 2007; 33: 344-7.

26. Dullabh HD, Slabbert JC and Becker PJ. Partial denture prosthodontic procedures employed by practising graduates of the University of the Witwatersrand, Johannesburg. SADJ. 1993; 48: 129 -4.

27. Owen CP. Fundamental of Removable Partial Dentures. $2^{\text {nd }}$ ed. Landsdowne: Cape Town University, Cape Town Press, 2000; 25.

28. Mothopi-Peri M, Owen CP. Guide-Plane Retention in Designing Removable Partial Dentures. Int J Prosthodont 2018; 31: $145-8$.

29. McCord JF, Grey NJ, Winstanley RB, Johnson A. A clinical overview of removable prostheses: 3. Principles of design for removable partial dentures. Dent Update. 2002 Dec; 29: 474-81.

30. Goodacre CJ, 1987. A dislodged and swallowed unilateral removable partial denture. J Prosthet Dent. 1987; 58: 124-5.

\section{Do the CPD questionnaire on page 275}

The Continuous Professional Development (CPD) section provides for twenty general questions and five ethics questions. The section provides members with a valuable source of CPD points whilst also achieving the objective of CPD, to assure continuing education. The importance of continuing professional development should not be underestimated, it is a career-long obligation for practicing professionals.

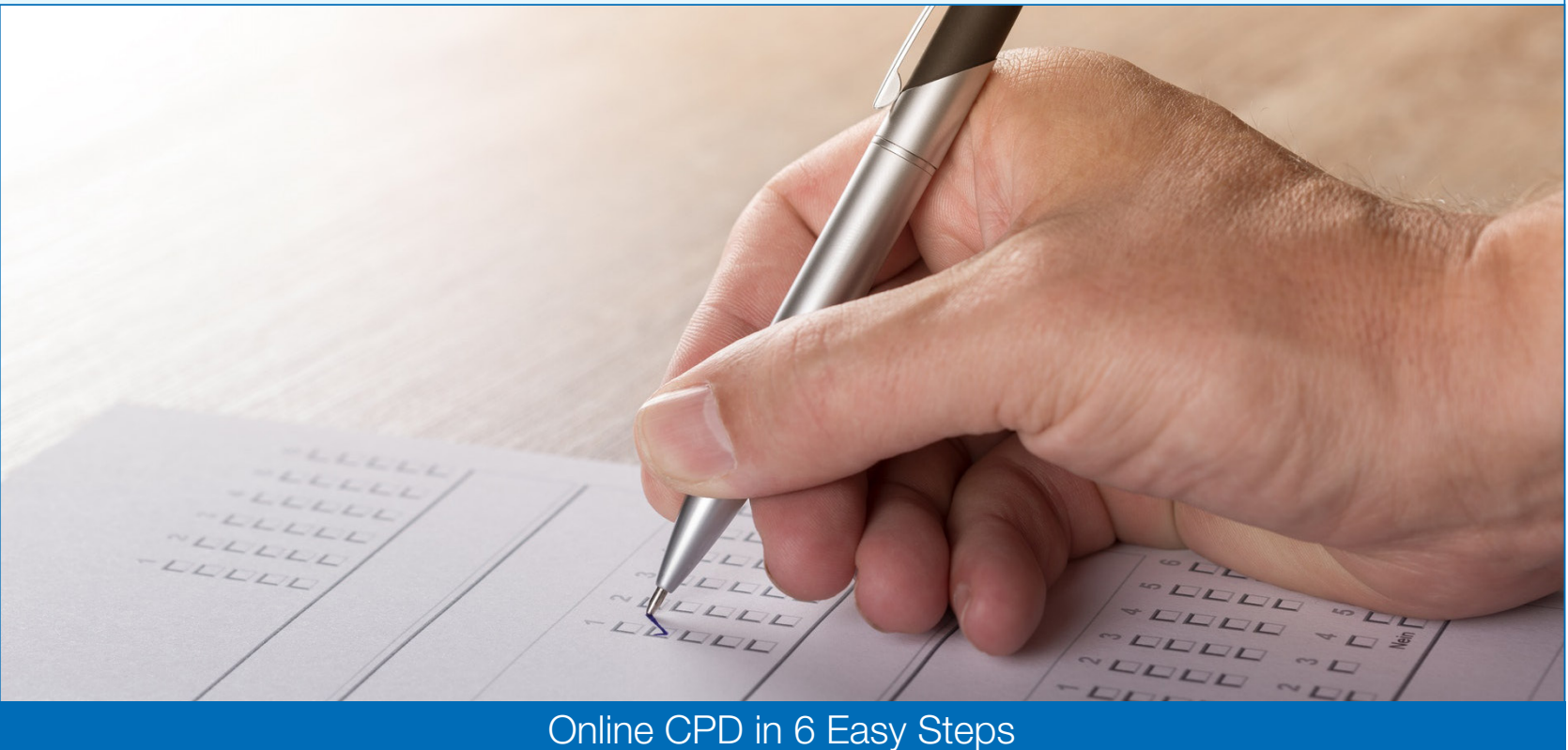

1 Go to the SADA website www.sada.co.za

2 Log into the 'member only' section with your unique SADA username and password.

3 Select the CPD navigation tab.

4 Select the questionnaire that you wish to complete.

5 Enter your multiple choice answers. Please note that you have two attempts to obtain at least $70 \%$.

6 View and print your CPD certificate. 